\title{
Learning and Survival of Newly Generated Neurons: When Time Matters
}

\author{
Aurélie Mouret, ${ }^{1}$ Gilles Gheusi, ${ }^{1}$ Marie-Madeleine Gabellec, ${ }^{1 \star}$ Fabrice de Chaumont, ${ }^{2 \star}$ Jean-Christophe Olivo-Marin, ${ }^{2}$ \\ and Pierre-Marie Lledo ${ }^{1}$ \\ ${ }^{1}$ Perception and Memory Laboratory, Centre National de la Recherche Scientifique (CNRS) Unité de Recherche Associée (URA) 2182, Institut Pasteur, \\ 75015 Paris, France, and ${ }^{2}$ Unité Analyse d'Images Quantitative, CNRS URA 2582, Institut Pasteur, 75015 Paris, France
}

\begin{abstract}
New interneurons are continually added to the olfactory bulb $(\mathrm{OB})$, the first central relay for processing olfactory information, throughout life. It remains unknown how these adult-generated interneurons integrate into preexisting networks or die. We used immunohistochemical approaches to quantify adult neurogenesis in mice subjected to olfactory training. We identified a critical period in the life of an adult-generated $\mathrm{OB}$ interneuron, during which learning triggers distinct consequences. Using a discrimination learning task performed at various times after the birth of new interneurons, we found that olfactory training could increase, decrease, or have no effect on the number of surviving newly generated neurons. Cell survival and elimination depend on both the age of the cell and its location within the granule cell layer. This study provides new insight into the contribution of the newly generated interneurons to $0 B$ function. It demonstrates that neuronal elimination is an active process, rather than a simple consequence of nonuse.
\end{abstract}

Key words: adult neurogenesis; GABA; olfactory bulb; interneurons; discrimination; plasticity

\section{Introduction}

The olfactory bulb $(\mathrm{OB})$ receives newly generated interneurons throughout life in rodents (Altman and Das, 1965), at an estimated rate of several thousand new neurons per day (Lledo et al., 2006). These newcomers originate from a periventricular region called the subventricular zone (SVZ), migrate along the rostral migratory stream (RMS), and differentiate principally into periglomerular cells and granule cells (GCs), the two main local interneuron populations of the OB circuit (Lledo et al., 2008).

At first glance, adult neurogenesis seems to have no purpose, because only a subset of newly generated interneurons survive, a substantial proportion of the new cells having only a short existence (Petreanu and Alvarez-Buylla, 2002; Winner et al., 2002; Lagace et al., 2007; Ninkovic et al., 2007). Experiments based on use of bromodeoxyuridine $(\mathrm{BrdU})$ and $3^{\mathrm{H}}$-thymidine have shown that almost one-half of the new GCs produced are eliminated within a few weeks in adult rodent OBs (Petreanu and Alvarez-Buylla, 2002; Winner et al., 2002). This high turnover contrasts with the situation in early postnatal periods, in which new neurons are continually added to the developing $\mathrm{OB}$ as longlasting building blocks (Lemasson et al., 2005). Adult-generated

\footnotetext{
Received June 26, 2008; revised Aug. 5, 2008; accepted Sept. 8, 2008.

P.-M.L. is supported by the Fondation pour la Recherche Médicale, Ecole des Neurosciences de Paris, Fédération pour la Recherche sur le Cerveau, Agence Nationale de la Recherche (ANR-2007-SEST-15 03), and the Groupe lonis. The Perception and Memory Laboratory is part of the Network of European Neuroscience Institutes (ENI-NET; LSHM(T-2005-019063). We thank Alix Birchenall and Inmaculada Ortega-Perez for technical assistance with histology. *M.-M.G. and F.d.C. contributed equally to this work.

Correspondence should be addressed to Pierre-Marie Lledo, Perception and Memory Laboratory, Institut Pasteur,

25 rue du Dr. Roux, 75015 Paris Cedex 15, France. E-mail: pmlledo@pasteur.fr.

DOI:10.1523/JNEUROSCI.2954-08.2008

Copyright $\odot 2008$ Society for Neuroscience $\quad 0270-6474 / 08 / 2811511-06 \$ 15.00 / 0$
}

OB neurons are more labile, replaceable functional units, subject to unknown selective events. Their survival rate may depend on the experience of the animal, because adult circuits can modify the morphological and functional properties of newborn neurons through activity-dependent processes. For instance, the deprivation of olfactory sensory inputs delays maturation and impairs the survival of new OB interneurons (Petreanu and AlvarezBuylla, 2002; Winner et al., 2002). In contrast, long-term exposure to a rich range of odors increases the survival of newly generated interneurons (Rochefort et al., 2002) and transiently improves odor memory (Rochefort and Lledo, 2005), suggesting a potential role for adult neurogenesis in olfactory memory.

The nature of the neuronal activity required to regulate adult neurogenesis was recently investigated. More 30-d-old GCs survived in mice that had learned to discriminate odorants than in the control mice (Alonso et al., 2006). Because olfactory deprivation dramatically decreases the survival rate of 14- to 28-d-old neurons (Yamaguchi and Mori, 2005), it has been suggested that sensory inputs in a learning context may play a key role in controlling the survival of newcomers (Alonso et al., 2006). However, it remains debated whether the benefit of discrimination learning could be extended to younger and older interneurons generated during adulthood. Learning to discriminate between two odorants was shown to decrease the survival of 45-d-old neurons in one study (Mandairon et al., 2006), in sharp contrast to our previous study (Alonso et al., 2006). Given the difference in the age of newly generated neurons analyzed in the two studies, it is possible that learning affects new neurons differently as a function of their maturation status. We tested this hypothesis in this study. We analyzed the effects of learning on the lifespan of newly generated neurons of different ages and found that learning promoted the survival of immature new neurons and the elimination 
of more mature neurons. In contrast, learning had no effect on the new neurons just reaching the $\mathrm{OB}$ circuit and those that were fully mature. Surprisingly, changes in neuronal survival concerned only a subset of the entire population of GCs. We conclude that olfactory learning induces cascades of stabilization/ elimination events that lead to complex regulation of the number of surviving newly generated neurons in the adult OB circuit.

\section{Materials and Methods}

Mice. Two-month-old male C57BL/6J mice (Janvier) were used throughout this study. For behavioral experiments, all animals (trained and controls) were given restricted access to water. Control animals were subjected to the same protocol as the trained group, except for odorant exposure (see supplemental Methods, available at www.jneurosci.org as supplemental material). All experimental procedures were performed in accordance with the Charter of Fundamental Rights of the European Union (2000/C 364/01), the European Communities Council Directive of 24 November 1986 (86/609/EEC), and European Union guidelines, and were reviewed and approved by our Institutional Animal Welfare Committee. In particular, all efforts were made to minimize animal suffering and the number of animals used.

BrdU injections. Mice were injected intraperitoneally with BrdU (75 $\mathrm{mg} / \mathrm{kg}$, Sigma-Aldrich). For neuronal survival studies, mice received four injections, $2 \mathrm{~h}$ apart, on the same day, and were systematically perfused $2 \mathrm{~d}$ after the last training session. To study neuronal proliferation, mice were injected once with $\mathrm{BrdU}$ and killed $2 \mathrm{~h}$ later.

Immunohistochemistry. Immunostaining was performed on $40 \mu \mathrm{m}$ coronal or sagittal free-floating sections, and slices were processed for BrdU, BrdU/NeuN, and doublecortin (DCX) labeling (see supplemental Methods, available at www.jneurosci.org as supplemental material).

Image acquisition. For light microscopy (BrdU staining), a $20 \times$ objective was used to reconstruct images of each section (Compix Imaging; Hamamatsu Photonics). For the acquisition of fluorescence images (BrdU/ NeuN and DCX/DAPI staining), a microscope (Zeiss) equipped with an Apotome and Axiovision 4.6 software was used $(25 \times$ objective or $63 \times$ objective) (see supplemental Methods, available at www.jneurosci.org as supplemental material). Sections were coded blind until the completion of data analysis.

Analysis of $\mathrm{BrdU}^{+}$and $\mathrm{DCX}^{+}$cells. BrdU ${ }^{+}$cells were counted automatically with a specific computer program (see supplemental Methods, available at www.jneurosci.org as supplemental material). For each animal, counts were made for one in every three coronal sections of the OB. The internal and external borders of the glomerular (GL), external plexiform (EPL), and granule cell (GCL) layers and the border of the rostral migratory stream of the $\mathrm{OB}\left(\mathrm{RMS}_{\mathrm{OB}}\right)$ were drawn. For sagittal sections, all consecutive slices containing a clear SVZ and RMS were counted. Interactive drawings of the SVZ, the vertical RMS (RMSv), and the horizontal RMS (RMSh) were produced. The program then counted the cells detected in the various zones. Values were given as $\mathrm{BrdU}^{+}$cell density (number of positive cells per square millimeter). For DCX staining, the same software was used to quantify the area covered by $\mathrm{DCX}^{+}$cells.

Behavioral apparatus, training, and odorants. Partially water-deprived mice were trained using an operant conditioning go/no-go paradigm in home-made computer-controlled olfactometers. They were trained to respond to the presence of an odor (positive stimulus: $\mathrm{S}+$ ) by licking the water delivery tube (located within the odor sampling port) and to refrain from responding to the presence of another odor (or of clean air) (negative stimulus: $\mathrm{S}-$ ). In each trial, a single stimulus ( $\mathrm{S}+$ or $\mathrm{S}-$ ) was presented, and the percentage of correct responses was determined for each block of 20 trials. All mice underwent a session of 10 blocks (200 trials) per day, during the training period. All the odorants used were diluted in odorless mineral oil, and the odorant concentrations are given as the dilution of the odorant in the saturator tubes (see supplemental Methods, available at www.jneurosci.org as supplemental material). To check that the reported effects are attributed to olfactory learning only, we ensured that the olfactometer training procedures did not per se alter neuronal survival (see supplemental Data 1 and Fig. S1, available at www.jneurosci.org as supplemental material).
Data analysis. For each zone studied, mean $\mathrm{BrdU}^{+}$cell density was calculated and averaged for each group. In some experiments, a normalized cell density was calculated for groups of trained animals with respect to their own control group (see supplemental Methods, available at www.jneurosci.org as supplemental material).

\section{Results \\ Olfactory discrimination learning increases neuronal survival in the $\mathrm{OB}$}

We investigated the consequences of learning on adult neurogenesis, by labeling newly generated neurons with BrdU and counting labeled cells in the OB $24 \mathrm{~d}$ later (Fig. 1 A). Eight days after BrdU injection, mice underwent 2 weeks of training in go/no-go odor discrimination tasks, in which a correct response to one odorant $(\mathrm{S}+)$ was rewarded by water (Fig. $1 B$ ). When a new odorant (or pair of odorants) was presented, animals initially had a performance similar to that expected based on chance (i.e., $50 \%$ correct responses), but gradually improved to attain levels of $>85 \%$. In subsequent sessions, mice more rapidly achieved this performance criterion (Fig. 1C). All mice learned the different tasks and achieved the performance criterion during the first few sessions. By quantifying $\mathrm{BrdU}^{+}$cells in the $\mathrm{OB}$, we investigated whether adult neurogenesis might be affected by this training (Fig. 1D). Consistent with previous reports, new cell density was highest in the GCL (Alonso et al., 2006). Interestingly, the number of newly generated cells in this layer increased with learning $\left(t_{(10)}=3.55, p=0.005\right)$ (Fig. $\left.1 E, F\right)$. In contrast, the density of $\mathrm{BrdU}^{+}$cells remained constant in both the GL $\left(t_{(10)}=0.74, p=\right.$ $0.475)$ and $\operatorname{EPL}\left(t_{(10)}=1.51, p=0.161\right)$. We investigated whether the newly generated cells had a neuronal phenotype, by analyzing the coexpression of BrdU with the neuronal marker NeuN. The percentage of $\mathrm{BrdU}^{+}$cells also labeled with NeuN was high in the control group $(>90 \% ; n=6)$ and was not affected by training $\left(t_{(10)}=0.06, p=0.952\right.$ ) (Fig. 1G). Thus, the increase in $\mathrm{BrdU}^{+}$ cell density in the GCL of trained mice mainly concerned a neuronal population.

\section{Learning increases both the survival rate and elimination of newborn neurons}

We characterized the time course of neuronal survival in the $\mathrm{GCL}$, by quantifying $\mathrm{BrdU}^{+}$cells at various time points after BrdU injections. The density of $\mathrm{BrdU}^{+}$cells differed considerably between days (Fig. $2 A$ ). It rapidly decreased between day 14 $(\mathrm{D} 14)$ and D30 $\left(t_{(12)}=13.34, p<0.001\right)$ and then decreased more slowly between D30 and D65 $\left(t_{(12)}=5.15, p<0.001\right)$ (Fig. $2 A)$. Thus, there was an early, dramatic wave of cell elimination between 2 and 4 weeks after cell generation.

Then, we investigated whether training affected this survival rate. At different time points after BrdU administration, mice were trained to recognize a single odorant (e.g., linalool) during a 1 week training (Fig. $2 \mathrm{~B}$ ). The concentration of this odorant was decreased every day (Fig. 2C). We found that olfactory learning affected neuronal survival (Fig. 2D). The number of 11-d-old neurons and of fully mature neurons ( $65 \mathrm{~d}$ old $)$ was not affected by learning $\left(t_{(12)}=0.26, p=0.79\right.$ and $t_{(12)}=0.17, p=0.86$, respectively), whereas the survival rate of 18-, 23-, and 30-d-old neurons was significantly enhanced by learning $\left(t_{(10)}=4.31, p=\right.$ $0.001 ; t_{(12)}=4.12, p=0.001$; and $t_{(10)}=8.31, p<0.001$, respectively). In contrast, the population of 38 -d-old neurons was dramatically decreased by learning $\left(t_{(12)}=4.47, p<0.001\right)$. We detected no change in apoptosis in the $\mathrm{OB}$ with training (data not shown), possibly because of the opposing effects of learning on neuronal survival, and because of differences in the kinetics of the 
A

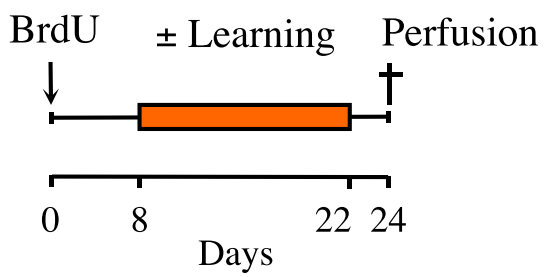

C

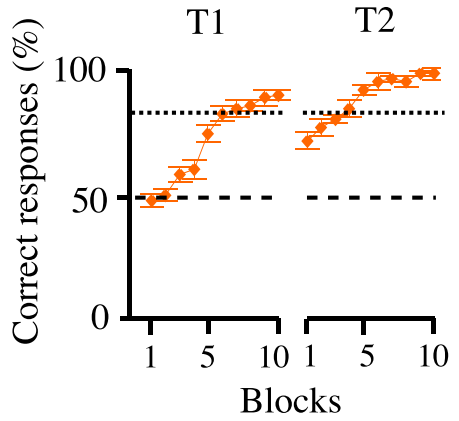

B

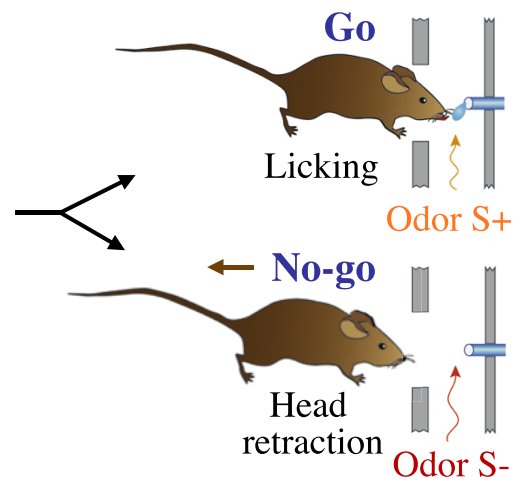

D

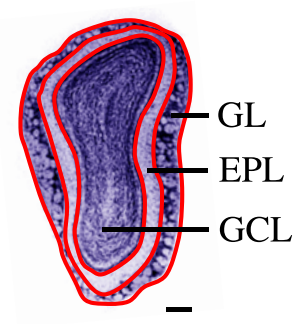

$\mathbf{E}$

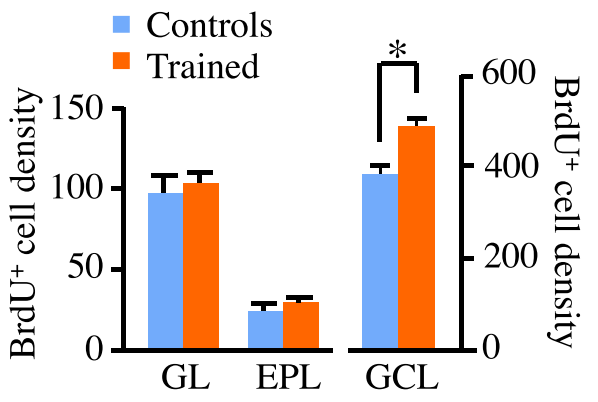

$\mathbf{F}$

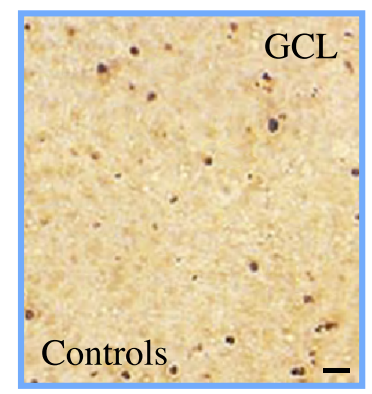

BrdU

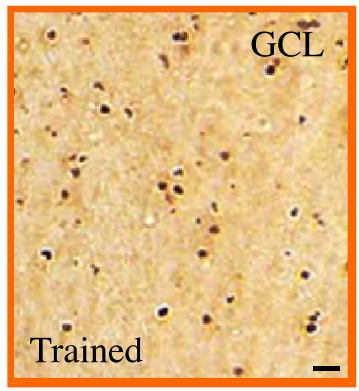

G

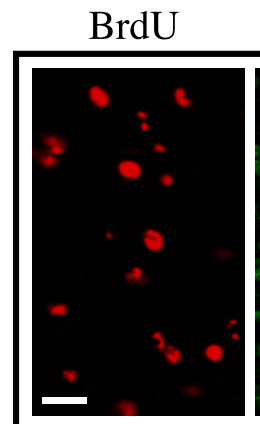

NeuN

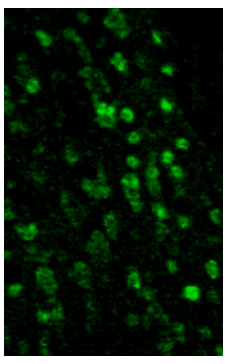

Merged

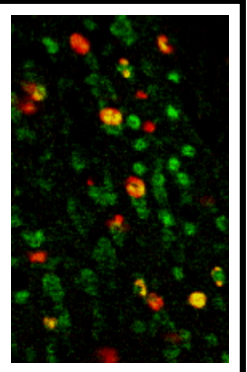

Figure 1. Two weeks of olfactory discrimination learning increases neuronal survival in the GCL but not in the EPL and GL. $\boldsymbol{A}$, New cells were labeled with BrdU, and their survival was evaluated $24 \mathrm{~d}$ later. Trained mice underwent discrimination learning when BrdU ${ }^{+}$cells were between 8 and $22 \mathrm{~d}$ old $(n=6)$. $\boldsymbol{B}$, Go/no-go procedure. $\boldsymbol{C}$, Mean percentage of correct responses in each block of the first $2 \mathrm{~d}$ (T1 and T2) of training $(n=6) . S+$ was amyl acetate, and S - was mineral oil. The dashed line represents chance level (50\%), and the dotted line represents performance criterion (85\%). D, A coronal section of the left OB stained with the nuclear dye T0T03 to visualize the different layers. $\boldsymbol{E}, \mathrm{BrdU}{ }^{+}$cell density in the $\mathrm{GL}, \mathrm{EPL}, \mathrm{and} \mathrm{GCL}$ after training. ${ }^{*} p=0.005(n=6) . \boldsymbol{F}$, Representative images showing BrdU ${ }^{+}$cells in the $\mathrm{GCL}$ of control and trained animals $24 \mathrm{~d}$ after BrdU administration. G, Double-labeled cells in the $\mathrm{GCL} 24 \mathrm{~d}$ after BrdU administration, showing the colocalization of BrdU (red) with NeuN (green). Scale bars: $\boldsymbol{D}, 100 \mu \mathrm{m} ; \boldsymbol{F}, \mathbf{G}, 15 \mu \mathrm{m}$. Error bars indicate the SEM.

two phenomena. We checked also that the changes in newly generated cell numbers were independent of the odorant quality and could be observed all along the rostrocaudal axis of the OB (see also supplemental Data 2 and Fig. S2, available at www. jneurosci.org as supplemental material).

\section{Learning does not alter proliferation or migration but does} modify the spatial distribution of adult-generated interneurons The larger number of new interneurons in the OB after learning may be associated with an increase in the rate of cell proliferation or neuroblast migration. We investigated these possibilities by administrating a BrdU injection to mice after a week of training (identical to the one described in Fig. 2C), and killing them $2 \mathrm{~h}$ later. In trained and control mice, BrdU labeling revealed the presence of dividing precursor cells along the entire SVZ-OB pathway (Fig. $3 A$ ). However, no difference in $\mathrm{BrdU}^{+}$cell density within the SVZ $\left(t_{(8)}=0.55, p=0.60\right)$, the RMSv $\left(t_{(8)}=1.07, p=\right.$ $0.32)$, and the RMSh $\left(t_{(8)}=1.49, p=0.17\right)$ was found between control and trained groups (Fig. 3B). Migrating neuroblasts with classic bipolar shapes were also visualized by DCX immunostaining in the RMS (Fig. 3C). Control and trained groups did not differ in the size of the SVZ $\left(t_{(8)}=0.398, p=0.7\right)$, the RMSv $\left(t_{(8)}\right.$ $=1.70, p=0.126)$, or the RMSh $\left(t_{(8)}=0.45, p=0.45\right)($ Fig. $3 D)$.

GCs generated during adulthood may be subdivided into distinct populations. Superficial GCs, the dendrites of which target primarily the superficial lamina of the EPL, are thought to establish synapses with tufted cells (Shepherd et al., 2004), whereas deep GCs mostly contact the dendrites of mitral cells in the deep lamina of the EPL (Mori et al., 1983) (Fig. 4A). We investigated whether the changes induced by learning were homogeneous throughout the GCL, by focusing on 30- and 38-d-old neurons (Fig. $4 B$ ). We analyzed the distribution of these newly generated cells, using a precise counting methodology (Fig. 4C). As previously reported (Lemasson et al., 2005), the density of new neurons was higher in deeper portions of the GCL (ring effect: Fig. $4 D, F_{(9,108)}=290.83, p<0.001$; Fig. $4 E, F_{(9,108)}=111.625, p<$ 
A

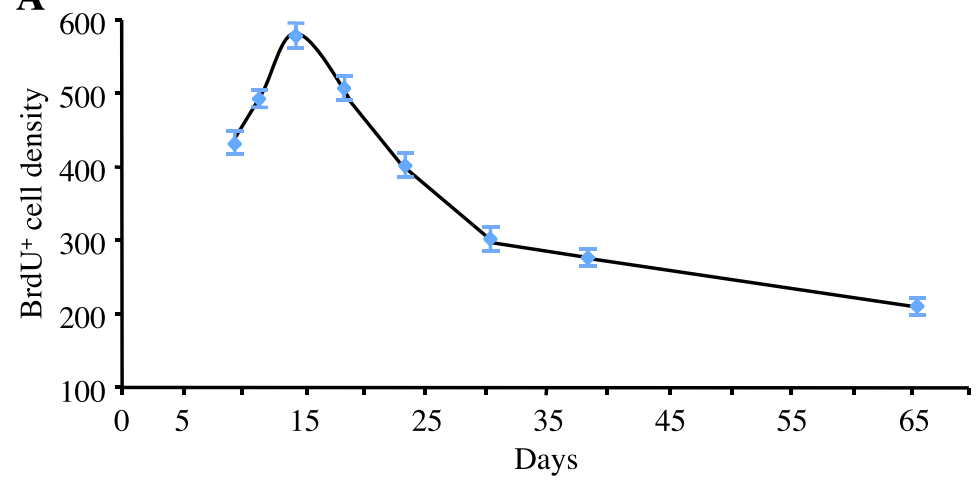

$\mathbf{C}$

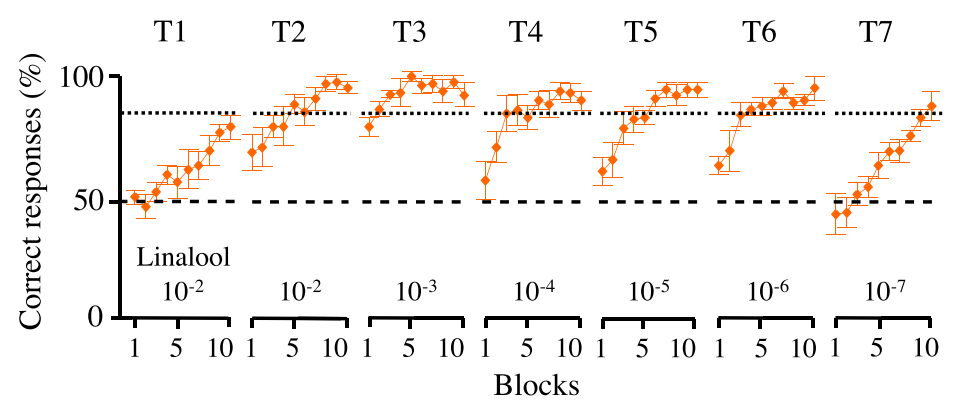

B

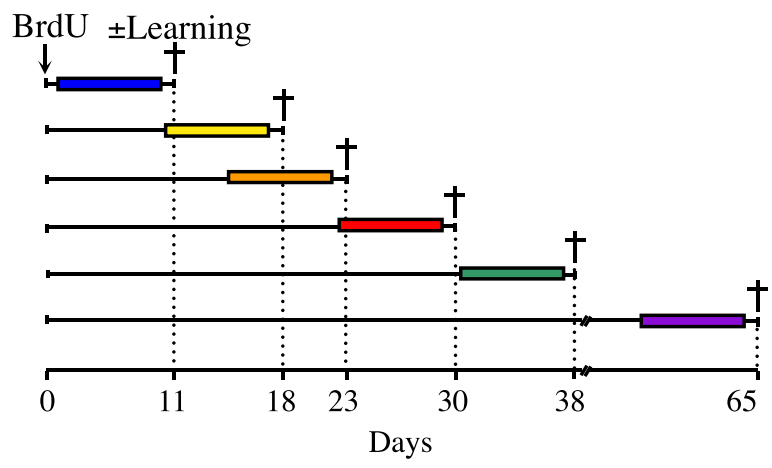

D

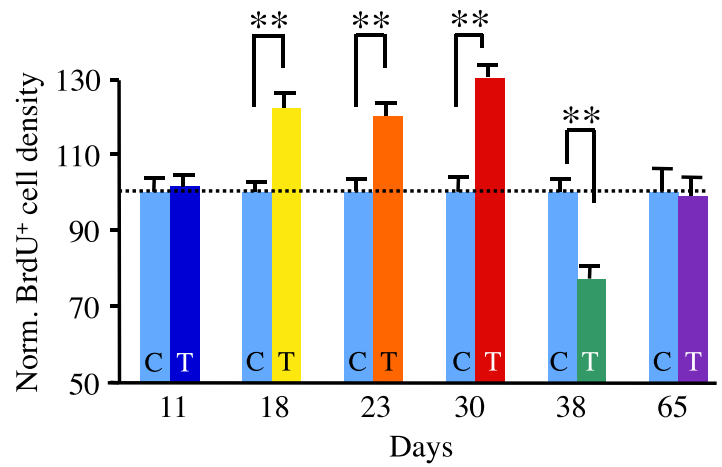

Figure 2. Opposite effects of olfactory learning on neuronal survival. $\boldsymbol{A}$, Changes in BrdU ${ }^{+}$cell density over time in naive animals after BrdU administration (day 0$)(n=6-7) . \boldsymbol{B}$, New cells were labeled with BrdU, and their survival was evaluated 11, 18,23,30,38, and $65 \mathrm{~d}$ later. Nine days before perfusion, trained mice were subjected to 1 week of olfactory training with a single odorant $(n=6-7)$. C, Mean percentage of correct responses in each block of the $7 \mathrm{~d}$ (T1-T7) of training $(n=6-7)$. S + was linalool (decreasing concentrations), and S - was mineral oil. The dashed line represents chance level (50\%), and the dotted line represents performance criterion (85\%). D, BrdU ${ }^{+}$cell density in the GCL after training. Values were normalized with respect to controls. C, Control mice, $\mathrm{T}$, trained mice. ${ }^{* *} p \leq 0.001(n=6-7)$. Error bars indicate the SEM.

0.001). We also confirmed that the distribution of the entire population of BrdU ${ }^{+}$ cells showed a pronounced effect of learning both on cell survival (group effect: Fig. $\left.4 D, F_{(2,12)}=6.22, p=0.014\right)$ and cell elimination (group effect: Fig. $4 E, F_{(2,12)}=$ $11.51, p=0.002$ ). This effect depended on the position of the cells in the GCL (interaction ring $\times$ group: Fig. $4 D, F_{(18,108)}=$ 6.30, $p<0.001$; Fig. $4 E, F_{(18,108)}=8.06$, $p<0.001)$. It was more marked in the deeper parts of the GCL (group effect for deep cells: Fig. $4 D, F_{(2,12)}=11.01, p=$ 0.001 ; Fig. $\left.4 E, F_{(2,12)}=17.27, p<0.001\right)$ than close to the mitral cell layer (group effect for superficial cells: Fig. $4 D, F_{(2,12)}=$ 1.70, $p=0.22$; Fig. $4 E, F_{(2,12)}=1.92, p=$ $0.18)$. Thus, we conclude that newly generated GCs sensitive to olfactory learning are preferentially located in the internal portion of the GCL.

\section{Discussion}

Active neurogenesis occurs in the adult $\mathrm{OB}$, but only a small percentage of newborn interneurons survive. This turnover of interneurons allows for a unique opportunity to get insight into neurogenesis as it relates to behavior. Here, we have analyzed adult neurogenesis in relation to the learning of an odor discrimination task. We report

C ventricle, Ctx, cortex.
A
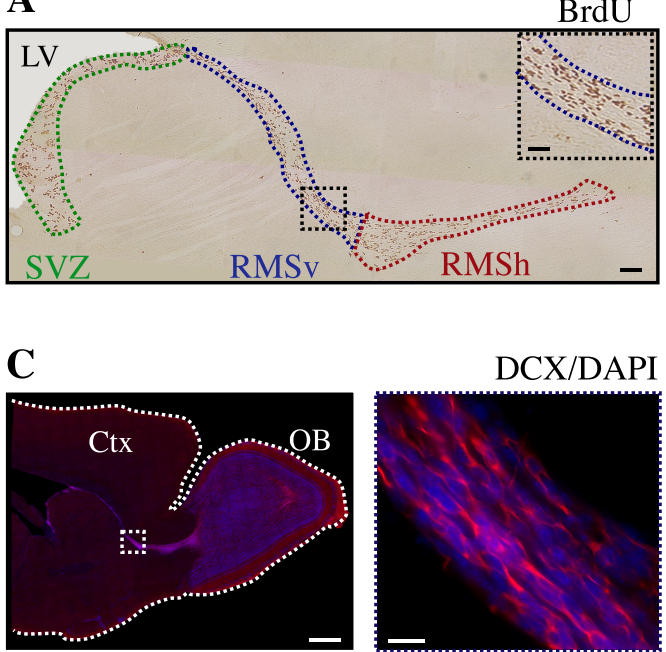

Figure 3. Olfactory learning has no effect on the first steps of neurogenesis. $A, A$ sagittal section of the forebrain showing the different neurogenic zones labeled with BrdU. Inset, Higher magnification of BrdU staining. B, BrdU ${ }^{+}$cell density in the SVZ, RMSv, and RMSh after training $(n=5)$. C, A sagittal section of the forebrain showing the SVZ-0B pathway labeled with DCX (red) and DAPI (blue) (left) and a higher magnification of the DCX/DAPI staining (right). $D$, Area of the SVZ, RMSv, and RMSh after training $(n=5)$. Scale bars: $\boldsymbol{A}, 250 \mu \mathrm{m} ; \boldsymbol{A}$, inset, $20 \mu \mathrm{m} ; \boldsymbol{C}($ left), $1 \mathrm{~mm} ; \boldsymbol{C}$ (right), $10 \mu \mathrm{m}$. Error bars indicate the SEM. LV, Lateral

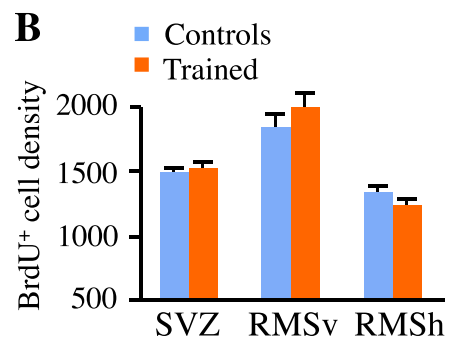

D $\quad$ Controls
Trained

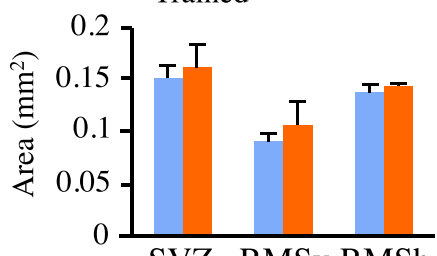

SVZ RMSv RMSh successive periods of no effect, increased survival, decreased survival, and no change with regard to the maturation of newborn interneurons. Remarkably, only newly generated interneurons from the deep portion of the GCL are sensitive to learning. 
A

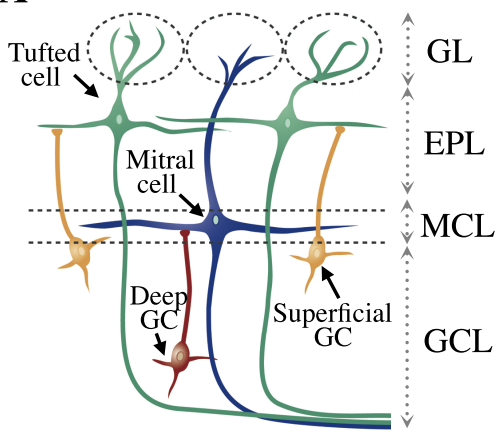

B

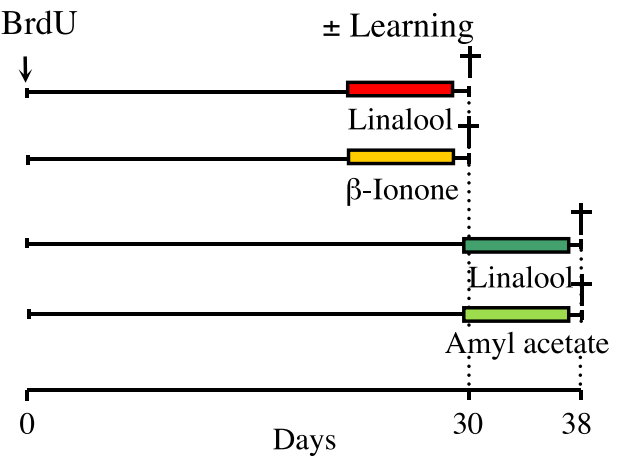

C

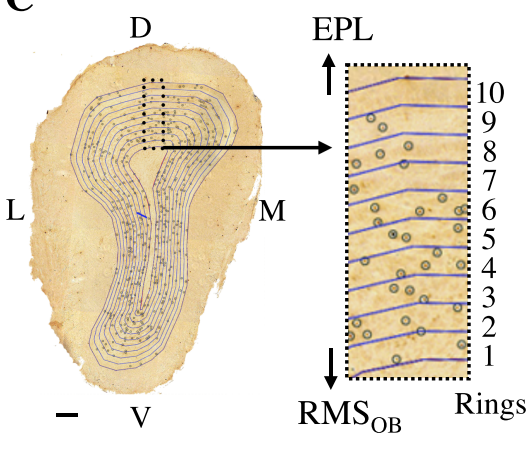

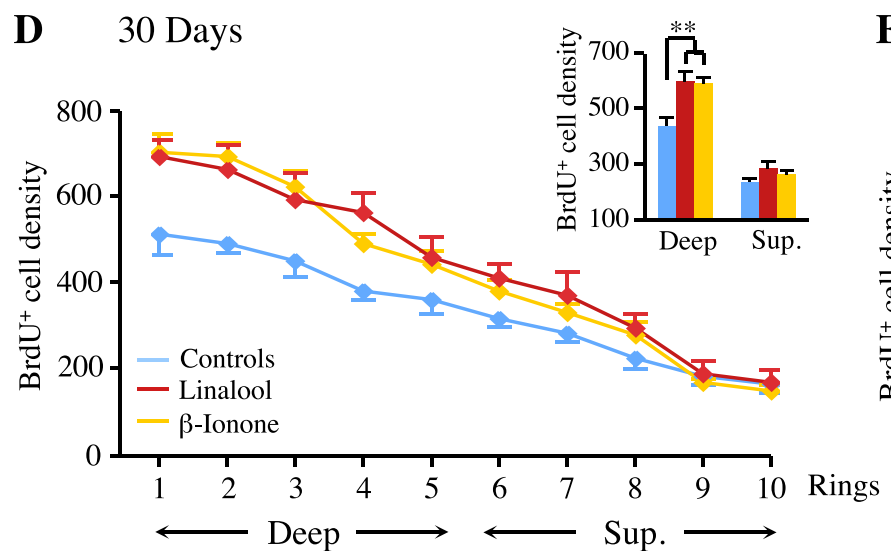

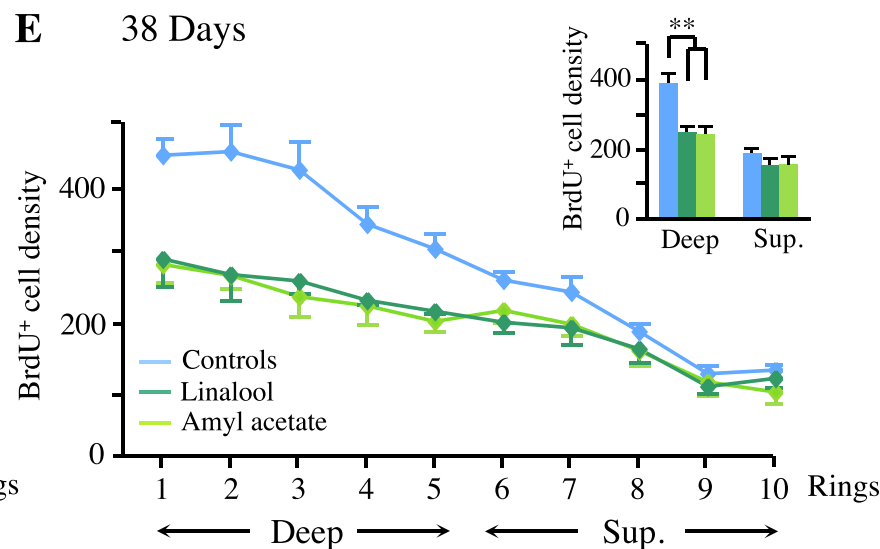

Figure 4. Newly generated deep GCs are more sensitive to olfactory learning than superficial GCs. $A, A$ schematic diagram of the wiring of the $0 B$, showing two populations of GCs as a function of location within the $\mathrm{GCL}$. $\boldsymbol{B}$, New cells were labeled with BrdU, and their survival was evaluated 30 and 38 d later. Nine days before perfusion, trained mice underwent olfactory training $(n=5)$. $S+$ was linalool, $\beta$-ionone, or amyl acetate, and $S$ - was mineral oil. C, Quantification of BrdU ${ }^{+}$cell distribution along the entire internal- external axis of the $G C L$ (D, dorsal; $L$, lateral; $V$, ventral; $\mathrm{M}$, medial). D, Distribution of BrdU ${ }^{+}$cell density along the internal- external axis of the $\mathrm{GCL}$, after training, for animals perfused $30 \mathrm{~d}$ after BrdU administration. Inset, Comparison of BrdU ${ }^{+}$cell density for deep (rings 1-5) and superficial (rings $6-10) \mathrm{GCs}$. ${ }^{* *} p=0.001(n=5)$. $\boldsymbol{E}$, Distribution of BrdU ${ }^{+}$cell density along the internal- external axis of the $\mathrm{GCL}$, after training, for animals perfused $38 \mathrm{~d}$ after BrdU administration. Inset, Comparison of BrdU cell density for deep and superficial GCs. ${ }^{* *} p<0.001$ ( $\left.n=5\right)$. Scale bars, $100 \mu \mathrm{m}$. Error bars indicate the SEM. MCL, Mitral cell layer.

Recently, the impact of olfactory learning on newborn GCs survival has been investigated (Alonso et al., 2006; Mandairon et al., 2006). The two studies demonstrated that it was olfactory learning, and not the mere exposure to odorants, that changed OB neurogenesis. More 30-d-old newborn interneurons survived in the $\mathrm{OB}$ of mice that had learned to discriminate between two odorants (Alonso et al., 2006), whereas the opposite effect was seen in 45-d-old newborn GCs (Mandairon et al., 2006). Not only does the present study bring together these findings, but it also demonstrates that olfactory learning may distinctively modulate newborn neurons depending on their entire maturation status, from arrival to the bulb to complete synaptic integration.

Adult neurogenesis involves the proliferation, migration, differentiation, and survival or death of the newly generated cells (Zhao et al., 2008). Our behavioral task was performed at different times, between 2 and $56 \mathrm{~d}$ after BrdU administration. Theoretically, learning could have affected any of these processes. However, results based on short-term exposure to BrdU or DCX immunostaining indicated that neither cell proliferation nor migration was affected. This conclusion was supported by the lack of effect of learning on the cohort of 11-d-old cells, indicating that the major impact of olfactory learning resulted from changes in survival rate.

Studies of survival (from several days to weeks) showed that learning had a complex influence on neuronal turnover. Spatial learning has been shown to increase or decrease the survival of newborn hippocampal cells as a function of their age (Döbrössy et al., 2003; Leuner et al., 2004; Olariu et al., 2005). It has been suggested that cells of "inappropriate ages" (the youngest and least mature) tend to die during learning (Dupret et al., 2007) and that the enhancement of apoptosis may accentuate the survivalpromoting effect by eliminating newborn neurons that are not yet functional. This situation contrasts with $\mathrm{OB}$ neurogenesis, in which only cells aged between 18 and $30 \mathrm{~d}$ tended to survive longer after learning, with the older cells ( $38 \mathrm{~d}$ old) dying. It has been shown that mitogen-activated protein kinase (MAPK) in the GCs of the OB induces the production of $\mathrm{Bcl}-2$, an antiapoptotic protein (Miwa and Storm, 2005). Further studies are required to determine whether the learning-specific modification of cell survival requires MAPK activation and, if so, how the production of this protein can be so precisely regulated over time.

In adult life, the survival of newly generated neurons is critically regulated by the degree of sensory input, occurring only during a precise time window. Yamaguchi and Mori (2005) identified a sensitive period during which sensory experience strongly influences the survival of new GCs. This period (14-28 d after the formation of the cells) is consistent with our findings, in which enhanced survival was observed for 18- to 30-d-old neurons. Interestingly, at this time, the new cells begin to receive glutama- 
tergic synaptic contacts. This suggests that the survival of new GCs is experience dependent during the period of synapse formation. Once rescued from death by learning, they may remain for extended periods of time, perhaps even permanently. Further investigations are required to determine how long the learninginduced increase in neuronal survival lasts.

Evidence is accumulating for the existence of a relationship between adult neurogenesis and learning, but the precise role in learning of $\mathrm{OB}$ interneurons generated during adulthood remains a matter of debate. It has been suggested that newly generated neurons in the adult hippocampus must achieve full maturation before becoming functional and influencing behavior (Kempermann, 2008). However, the relative immaturity of the newly generated cells may be their most important feature, qualifying them for functions that more mature cells cannot achieve. Consistent with this hypothesis, it has been demonstrated that adult-born GCs are most responsive to new odors shortly after their synaptic integration (Magavi et al., 2005). As olfactory interneurons are continually generated, there is always a cohort of highly sensitive newcomers, providing a constant source of plasticity in the olfactory circuitry.

The functional implications of changes in the number and spatial distribution of newborn cells may be important for olfactory coding and learning. It has been suggested that olfactory coding is based on the temporal coherence of spatially distributed activity in the OB, largely under the control of GCs (Lledo et al., 2005), and the spatiotemporal output of the OB is known to be affected by learning (Fletcher and Wilson, 2003; Martin et al., 2004). The segregation of the GCs population into deep or superficial interneurons may indicate parallel, yet different inhibitory effects on projection neurons. The dendritic arbors of deep GCs are restricted to the deep EPL, in which they form synapses principally with mitral cell secondary dendrites (Fig. $4 A$ ). In contrast, the dendritic arbors of most superficial GCs are present in the superficial EPL, in which they synapse with tufted cell dendrites. Mitral and tufted cells project into different regions of the olfactory cortex, so these two populations of GCs may belong to separate $\mathrm{OB}$ microcircuits. The tufted-GCs synapses may mediate the low-threshold perception of odorants (Nagayama et al., 2004) through intrabulbar association. In contrast, mitral-GCs synapses may be important for odorant discrimination (Lledo and Lagier, 2006). Here, we demonstrate that adult-generated GCs sensitive to olfactory experience are located deep in the GCL. Therefore, we suggest that olfactory learning specifically fine tunes the survival of GABAergic interneurons dedicated to discrimination tasks. The bidirectional learning-induced neurogenic changes provide a means of adjusting odorant information processing, and support for the idea that new neurons make a unique contribution to the functioning of the adult OB network.

\section{References}

Alonso M, Viollet C, Gabellec MM, Meas-Yedid V, Olivo-Marin JC, Lledo PM (2006) Olfactory discrimination learning increases the survival of adult-born neurons in the olfactory bulb. J Neurosci 26:10508-10513.

Altman J, Das GD (1965) Post-natal origin of microneurones in the rat brain. Nature 207:953-956.

Döbrössy MD, Drapeau E, Aurousseau C, Le Moal M, Piazza PV, Abrous DN (2003) Differential effects of learning on neurogenesis: learning increases or decreases the number of newly born cells depending on their birth date. Mol Psychiatry 8:974-982.

Dupret D, Fabre A, Döbrössy MD, Panatier A, Rodríguez JJ, Lamarque S, Lemaire V, Oliet SH, Piazza PV, Abrous DN (2007) Spatial learning depends on both the addition and removal of new hippocampal neurons. PLoS Biol 5:e214.

Fletcher ML, Wilson DA (2003) Olfactory bulb mitral-tufted cell plasticity: odorant-specific tuning reflects previous odorant exposure. J Neurosci 23:6946-6955.

Kempermann G (2008) The neurogenic reserve hypothesis: what is adult hippocampal neurogenesis good for? Trends Neurosci 31:163-169.

Lagace DC, Whitman MC, Noonan MA, Ables JL, DeCarolis NA, Arguello AA, Donovan MH, Fischer SJ, Farnbauch LA, Beech RD, DiLeone RJ, Greer CA, Mandyam CD, Eisch AJ (2007) Dynamic contribution of nestin-expressing stem cells to adult neurogenesis. J Neurosci 27:12623-12629.

Lemasson M, Saghatelyan A, Olivo-Marin JC, Lledo PM (2005) Neonatal and adult neurogenesis provide two distinct populations of newborn neurons to the mouse olfactory bulb. J Neurosci 25:6816-6825.

Leuner B, Mendolia-Loffredo S, Kozorovitskiy Y, Samburg D, Gould E, Shors TJ (2004) Learning enhances the survival of new neurons beyond the time when the hippocampus is required for memory. J Neurosci 24:7477-7481.

Lledo PM, Lagier S (2006) Adjusting neurophysiological computations in the adult olfactory bulb. Semin Cell Dev Biol 17:443-453.

Lledo PM, Gheusi G, Vincent JD (2005) Information processing in the mammalian olfactory system. Physiol Rev 85:281-317.

Lledo PM, Merkle FT, Alvarez-Buylla A (2008) Origin and function of olfactory bulb interneuron diversity. Trends Neurosci 31:392-400.

Magavi SS, Mitchell BD, Szentirmai O, Carter BS, Macklis JD (2005) Adultborn and preexisting olfactory granule neurons undergo distinct experience-dependent modifications of their olfactory responses in vivo. J Neurosci 25:10729-10739.

Mandairon N, Sacquet J, Garcia S, Ravel N, Jourdan F, Didier A (2006) Neurogenic correlates of an olfactory discrimination task in the adult olfactory bulb. Eur J Neurosci 24:3578-3588.

Martin C, Gervais R, Hugues E, Messaoudi B, Ravel N (2004) Learning modulation of odor-induced oscillatory responses in the rat olfactory bulb: a correlate of odor recognition? J Neurosci 24:389-397.

Miwa N, Storm DR (2005) Odorant-induced activation of extracellular signalregulated kinase/mitogen-activated protein kinase in the olfactory bulb promotes survival of newly formed granule cells. J Neurosci 25:5404-5412.

Mori K, Kishi K, Ojima H (1983) Distribution of dendrites of mitral, displaced mitral, tufted, and granule cells in the rabbit olfactory bulb. J Comp Neurol 219:339-355.

Nagayama S, Takahashi YK, Yoshihara Y, Mori K (2004) Tufted cells differ in the decoding manner of odor maps in the rat olfactory bulb. J Neurophysiol 91:2532-2540.

Ninkovic J, Mori T, Götz M (2007) Distinct modes of neuron addition in adult mouse neurogenesis. J Neurosci 27:10906-10911.

Olariu A, Cleaver KM, Shore LE, Brewer MD, Cameron HA (2005) A natural form of learning can increase and decrease the survival of new neurons in the dentate gyrus. Hippocampus 15:750-762.

Petreanu L, Alvarez-Buylla A (2002) Maturation and death of adult-born olfactory bulb granule neurons: role of olfaction. J Neurosci 22:6106-6113.

Rochefort C, Lledo PM (2005) Short-term survival of newborn neurons in the adult olfactory bulb after exposure to a complex odor environment. Eur J Neurosci 22:2863-2870.

Rochefort C, Gheusi G, Vincent JD, Lledo PM (2002) Enriched odor exposure increases the number of newborn neurons in the adult olfactory bulb and improves odor memory. J Neurosci 22:2679-2689.

Shepherd GM, Wei RC, Greer CA (2004) Olfactory bulb. In: The synaptic organization of the brain, Ed 5 (Shepherd GM, ed), pp 165-216. New York: Oxford UP.

Winner B, Cooper-Kuhn CM, Aigner R, Winkler J, Kuhn HG (2002) Longterm survival and cell death of newly generated neurons in the adult rat olfactory bulb. Eur J Neurosci 16:1681-1689.

Yamaguchi M, Mori K (2005) Critical period for sensory experiencedependent survival of newly generated granule cells in the adult mouse olfactory bulb. Proc Natl Acad Sci U S A 102:9697-9702.

Zhao C, Deng W, Gage FH (2008) Mechanisms and functional implications of adult neurogenesis. Cell 132:645-660. 